\title{
Estimation of Residence Time in Continuous Flow Systems with Dynamics
}

\author{
Torbjörn Andersson \\ email: toand@isy.liu.se \\ Department of Electrical Engineering \\ Linköping University \\ S-581 83 Linköping, Sweden
}

\author{
Predrag Pucar \\ email: predrag@isy.liu.se \\ Department of Electrical Engineering \\ Linköping University \\ S-581 83 Linköping, Sweden
}

\begin{abstract}
A method for estimation of residence time in continuous flow systems with varying dynamics is presented. By resampling, i.e., choosing time instants different from the given sampling instants, and interpolation between measured data points, we obtain a continuous flow system with constant residence time expressed in the new resampled time vector. We assume the flow patterns in the systems are invariant. The new data set is then used for identification of parameters in a chosen model structure. From the identified model, the residence time is readily calculated and a procedure for that is briefly described. The presented method is readily extended to enable use in recursive identification. In that case, however, as an improvement of tracking ability of an ordinary recursive routine.

Keywords : System identification, residence time estimation, time-varying systems, variable flow and/or volume, continuous flow systems, recursive identification.
\end{abstract}

\section{Introduction}

In the process industry an often occurring problem is estimation of residence time for feed material components in vessels and tanks. The problem of identification and control of such systems has been addressed before. For alternative approaches of identification of above mentioned systems, we refer the reader to [6,7]. An interesting paper addressing the robust stability and robust performance of parameter-varying systems in the context of gain-scheduling is [8]. In [1] scaling with an independent quantity such as concentration, is used to decrease the time variability of a time variant, non-linear dynamic model. The concept is illustrated with the adaptive control of an anaerobic digester, where the independent variable is taken to be the cumulative amount of methane produced.

When a liquid element enters a vessel or tank the feed material in the element takes different ways through the flow system. In the sequel the more general name flow system will be used instead of vessel, tank etc. Although it is very natural to think about flow systems in terms of tanks and pipes, we would like to stress that the term is applicable to a wider range of applications such as rolling-mills, chemical industry, turbine control in power plants, systems where the system dynamics depends on a operating point etc. We define the residence time of the feed material as the expected time needed from entering at the input of the flow system to exiting at the output. The residence time $\bar{\tau}$ is composed of two parts, the pure time delay $\tau_{d e l}$ and the part due to the dynamics of the flow system $\tau_{d y n}$. The same notation is used in both the discrete and continuous time case. The 
division of the residence time into two parts is motivated by a simple model of a flow system. The flow system is modeled as a transportation part without mixing and a part where the material is mixed.

The volume and the flow through vessels and tanks, or more general the quantity influencing the dynamics of the continuous flow system, is time varying and that fact is an additional difficulty in the identification procedure. For example, the flow in an industrial process is subjected to changes both for random reasons, like for disturbances of various kind, and intentionally, when the production is increased or decreased. Likewise, the volume changes in a buffer vessel, and the purpose is to control the flow variations. When linear time invariant models are used in the identification procedure the time variability of the flow system will result in inaccurate linear time-invariant models, with unacceptably high variance of the parameters estimated. The method proposed in this paper solves the problem occurring in off-line identification (just mentioned), and also improves the result if a recursive identification routine is used to handle the time variability. The problem in recursive identification is the difficulty to track abrupt changes in residence time (and model parameters), which originate in the abrupt changes of the quantity influencing the dynamics of the flow system, and at the same time have a recursive identification routine which is insensitive to measurement noise. The method proposed in this report is exemplified on data from a part of a pulp production line, and the simplicity of the implementation of the same is illustrated in MATLAB, see Section 3.

Our method assumes that some relevant signals at the inflow and outflow of the flow system are measured. "Relevant" here means signals that are highly dependent on the material flowing through the flow system. The varying quantity influencing the dynamics, e.g. flow and/or volume, is also assumed to be simultaneously measured and recorded. The signals are, after processing by the method presented here, later used for identification of a chosen model structure from which it is straightforward to calculate the residence time. The identification method itself will not be treated in this report. For a thorough treatment of the topic of system identification we refer to $[3,5,9]$. The calculation of the residence time will be briefly addressed, and [2] will be extensively used.

The paper is organized as follows. In Section 2 preparing definitions are stated and calculation of residence time from obtained models is presented. Sections 3 and 4 contain the main contribution of this paper; the method for eliminating the quantity influencing the dynamics of the system in the calculation of residence time. Finally, in Section 5 the proposed method is tested on real data from a pulp process line.

\section{Definitions}

In this section we will introduce definitions needed later and we will mention identification routines used later in the paper. The calculation of residence time from the obtained model will also be presented.

Assume that a continuous flow system $g(t)$ with input $u$, and output $y$ is given. In other words the input-output relation is given by the convolution

$$
y(t)=\int_{\tau=0}^{\infty} g(t-\tau) u(\tau) d \tau .
$$

The input and output are signals that are highly dependent on the material flowing through the flow system, e.g., concentration, kappa number etc. We define the residence time $\tau_{d y n}$ 
as the center of gravity of the impulse response of the continuous flow system $g(t)$,

$$
\tau_{d y n}=\frac{\int_{0}^{\infty} t \cdot g(t) d t}{\int_{0}^{\infty} g(t) d t}
$$

Equation (1) can be interpreted in a probabilistic manner. Assume a "block" of liquid enters the flow system. The time it takes for the individual molecules to exit the system can be viewed as a random variable $\tau$ with the following density function

$$
f_{\tau}(t)=\frac{g(t)}{\int_{0}^{\infty} g(\sigma) d \sigma} .
$$

Obviously the residence time $\tau_{d y n}$ in this interpretation is the expected value of the time it takes for an molecule to pass through the flow system.

If we assume that the model of the continuous flow system is given, i.e., it has been identified by the identification routine used, an analytical way of calculating the residence time exists and is briefly presented below. For a detailed treatment we refer to [2].

From Equation (1) using the definition of the Laplace transform recognize $\tau_{d y n}$ as

$$
\tau_{d y n}=-\frac{G^{\prime}(0)}{G(0)}
$$

where $G(s)$ is the Laplace transform of the impulse response $g(t)$ and the numerator is the derivative of $G(s)$ with respect to $s$.

Using the rule of differentiation of a product it is easily checked that for a system $G(s)=G_{1}(s) G_{2}(s)$, i.e., two systems coupled in series, the residence time is

$$
\tau_{d y n}=\tau_{d y n_{1}}+\tau_{d y n_{2}}
$$

which is correct since the residence time of two continuous flow systems should be the sum of the respective residence times.

In discrete time we instead use the $z$-transform and the residence time, expressed in samples, is now defined as

$$
\tau_{d y n}=\frac{\sum_{k=0}^{\infty} k \cdot h(k)}{\sum_{k=0}^{\infty} h(k)} .
$$

The discrete time transfer function is defined as

$$
H(z)=\sum_{k=0}^{\infty} h(k) z^{-k},
$$

and it is easy to verify that in the discrete time case the residence time is

$$
\tau_{d y n}=-\frac{H^{\prime}(1)}{H(1)}
$$

The point is that parameters in $H(z)$,

$$
H(z)=\frac{B(z)}{A(z)}=z^{-k} \frac{b_{1}+b_{2} z^{-1}+\ldots+b_{m} z^{-m+1}}{1+a_{1} z^{-1}+\ldots+a_{n} z^{-n}}
$$

are delivered by the identification routine and the calculation in (2) is readily performed. In [2] the calculations are shown in detail.

There are mainly two ways to build a mathematical model of the process one is interested in. One way is to build a model that describes relations between input-output 
signals, based on the physical insight about the process inside the continuous flow system. That could be a number of differential equations describing the process. This can be very difficult due to lack of knowledge about what really is happening inside the system, e.g. strange flow patterns, old material suddenly falling off the walls of the vessel etc., and thus it is very difficult to mathematically describe the flow patterns inside the system.

The other way is to try to describe the connection between the input-output signals without using detailed knowledge about the system, at least not directly, but instead build linear "ready-made models", see [3], and estimate the unknown parameters. The knowledge about the system is not disregarded and it is used in the process of choosing the right model structure. The non-recursive identification method used is the well known least squares method, see [3]. The input-output relation can be written on the regression form

$$
y(t)=\varphi^{T}(t) \theta+e(t)
$$

where

$$
\varphi(t)=\left[\begin{array}{llll}
-y(t-T) \ldots-y\left(t-n_{a} \cdot T\right) & u\left(t-n_{k} \cdot T\right) \ldots u\left(t-\left(n_{k}+n_{b}-1\right) \cdot T\right)
\end{array}\right]^{T}
$$

and

$$
\theta=\left[\begin{array}{llllll}
a_{1} & \ldots & a_{n_{a}} & b_{1} & \ldots & b_{n_{b}}
\end{array}\right]^{T} .
$$

We see from (3) that the output signal is not affected by the input signal until $n_{k}$ samples later or in other words the pure time delay $\tau_{d e l}$ is $n_{k} \cdot T$ and has to be added to the calculation of the residence time (1) to obtain the total residence time $\bar{\tau}$.

We would like to point out that the calculation of residence time from the obtained model is only one possible use of the model. The model contains much more information which can be used for a variety of purposes (control, fault detection etc.).

For an exhaustive discussion on model structures and recursive identification we refer to $[3,5]$.

\section{$3 \quad$ Resampling}

In this paper we have used the technical computing environment MATLAB when exemplifying our method. The reason for including the MATLAB code in this paper is to illustrate one of the advantages of the presented algorithms; the implementational simplicity. Of course, any other programming language can be used. The code appearing in the paper are MATLAB m-files. For more information about MATLAB we refer to [4].

\subsection{Non-recursive case}

Assume that a continuous flow system is given. Further assume that the input-output signals are sampled with a fix sampling time $T$. Often continuous flow systems are modeled with time invariant dynamic systems and then a recursive algorithm takes care of the time variability, due to changes in the quantity influencing the dynamics. Here we look at a primarily non-recursive method, i.e., the input-output signals are preprocessed in such a way that a non-recursive method can be used. Since we are interested in the residence time, the input-output signals have to be highly dependent on the material flowing through the system.

Before stating the main results we will treat a special case of a time varying flow system. The objective is to show that the results presented later cover many cases in practice. 
Example: Assume that a perfectly mixed tank with volume $V$ is given. The differential equation describing the relation between the concentration at the input $c_{i n}$ and the concentration in the tank $c$ is the following

$$
\dot{c}(t)=-\frac{\chi(t)}{V} c(t)+\frac{\chi(t)}{V} c_{i n}(t) .
$$

We have assumed equal, but time varying, flow $\chi(t)$ into and out from the tank. The form of Equation (4) is typical for tank systems. Notice that the concentration in steady state in the tank always is equal to the concentration at the input (static gain equal to one).

Assume now that we allow a more general form of the time varying coefficients and denote them with $f(t)$. The coefficients are functions of time variable and constant quantities. In Equation (4), $f(t)$ is given by $f(t)=\chi(t) / V$.

The last generalization is that we allow the coefficients of the separate systems coupled in series and parallel to have different functions $f_{i}(t)$, where the functions $f_{i}$ are allowed to differ by a scaling factor, i.e., $f_{i}(t)=\alpha_{i} f(t)$. We will not investigate this special case, it is readily verified that the resampled system is time invariant by substitution in the proof of Theorem 3.1.

Definition 3.1 Let $G\left(p, f_{i}(t)\right), i=1 \ldots n$, denote a linear, time variant system, where $p$ is the differentiation operator $\frac{d}{d t}$. We define the dynamic influence functions (DIF), $f_{i}(t)$ as the functions that cause the time variability of the system.

If the functions $f_{i}$ are all equal we simply denote the function with $f(t)$.

In Theorem 3.1 the operator $q$ is the shift operator, i.e., $q y(t)=y(t+T)$ where $T$ is the sampling time.

Theorem 3.1 Assume $f_{1}=f_{2}=\ldots=f_{n}=f$, and let $G(p, f(t))$ have the following state space description

$$
\begin{aligned}
\dot{x}(t) & =f(t) A x(t)+f(t) B u(t) \\
y(t) & =C x(t),
\end{aligned}
$$

where $A, B$ and $C$ are constant system matrices.

Moreover, in physical systems an additional condition is often present, namely that the static gain is equal to one. For a tank system, for example, that means that all the material entering has to exit. Formally it can be expressed in the following way

$$
-C A^{-1} B=1
$$

Finally assume that the function $f(t)$ is a piecewise constant function. Then the continuous system can be non-uniformly sampled with the new sampling time $T_{t}$ in such way that there is no influence of the DIF in the discrete system $H(q)$, i.e., in

$$
x\left(t+T_{t}\right)=F\left(t, T_{t}\right) x(t)+G\left(t, T_{t}\right) u(t)
$$

the matrices $F\left(t, T_{t}\right)=F$ and $G\left(t, T_{t}\right)=G$ are constant.

Proof. Assume that the function $f(t)$ and the input $u(t)$ are constant on the time interval

$$
k T \leq t \leq k T+T_{t} .
$$


We will then have the solution to the system of differential equations

$$
x(t)=e^{A f(k T)(t-k T)} x(k T)+\int_{k T}^{t} e^{A \bar{f}(t-\tau)} B f(k T) u(k T) d \tau .
$$

To show that it is possible to remove the dependence of $f$ by choosing the sampling rate in an appropriate way, we have to make a series expansion of the non-constant part of the expression under the integral

$$
\begin{aligned}
x(t) & =e^{A f(k T)(t-k T)} x(k T)+B f(k T)\left(\int_{k T}^{t} e^{A f(k T)(t-\tau)} d \tau\right) u(k T) \\
& =e^{A f(k T)(t-k T)} x(k T)+B f(k T)\left(\int_{k T}^{t} \sum_{l=0}^{\infty} \frac{1}{l !}(A f(k T)(t-\tau))^{l} d \tau\right) u(k T) \\
& =e^{A f(k T)(t-k T)} x(k T)+B f(k T) \sum_{l=0}^{\infty} \frac{A^{l} f(k T)^{l}}{l !}\left(\int_{k T}^{t}(t-\tau)^{l} d \tau\right) u(k T) \\
& =e^{A f(k T)(t-k T)} x(k T)+B\left(\sum_{l=0}^{\infty} \frac{A^{l} f(k T)^{l+1}}{(l+1) !}(t-k T)^{l+1}\right) u(k T) .
\end{aligned}
$$

In the third equality the fact that the series is convergent is used. Finally let $t=k T+T_{t}$ and the sampled system is

$$
x\left(k T+T_{t}\right)=e^{A f(k T) T_{t}} x(k T)+B\left(\sum_{l=0}^{\infty} \frac{A^{l} f(k T)^{l+1}}{(l+1) !} T_{t}^{l+1}\right) u(k T) .
$$

Assume now that we choose the sampling increment, $T_{t}$, as

$$
T_{t}=\frac{\alpha}{f(t)} \quad \alpha \in \mathbf{R}
$$

The resulting system is then

$$
x\left(k T+T_{t}\right)=e^{A} \alpha x(k T)+B\left(\sum_{l=0}^{\infty} \frac{A^{l} \alpha^{l+1}}{(l+1 !)}\right) u(k T),
$$

which is independent of the DIF $f(t)$.

Remark: Note that the theorem is valid in the case of constant time delay. The simple solution to that is to adjust the input and output signal vectors before performing resampling. How variable delays can be treated in estimation of residence time is discussed in $[10]$.

In the example in this report it is assumed that the DIF is flow/volume $\left(f(t)=\frac{\text { flow }}{\text { volume }}\right)$. How to choose $f(\cdot)$ is an important issue. However, no general answer can be given since there are a variety of physical systems to which the presented idea could be applied, see Section 1 . The cause of the time variability, and the knowledge about the system one tries to model, are important.

When linear time invariant models are used in the off line identification procedure, the time variability of the flow system will result in inaccurate linear time-invariant models, with unacceptably high variance of the estimated parameters. Here we propose a two step method to improve the estimate of residence time; the first step being the removal 
of the influence of the DIF by resampling (treated later), and the second step being the usual identification method, for instance the least squares method referred to in Section 2, in combination with ARX-models. It will be exemplified that this two step method will improve the estimate of the residence time, if compared with an off-line method without any preprocessing (resampling) of data.

The resampling will be performed using the DIF in the following way. First we define the mean value of the DIF $\bar{f}$, which will be used in the sequel,

$$
\bar{f}=\frac{1}{N} \sum_{i=1}^{N} f(i) .
$$

We also define the sampling increment $\delta(i)$,

$$
\delta(i)=\bar{f} / f(i) .
$$

Recall that $T$ is the sampling time of the original system. How the resampling is performed depends on the sampling increment and it can be split up into two cases, namely when the DIF is larger respectively smaller than the mean value of the DIF. In the former case, i.e., $\delta(i)<1$, we resample, forming new time instants, more densely than the original sample instants. In the latter case, $\delta(i)>1$, we resample, forming new time instants, less densely than the original sample instants. In the case $\delta(i)=1$ we sample with the original sampling time $T$. If we denote the vector with the new time instants with $w$, we will have the new time instants $w(j)$ as $([x] \stackrel{\text { def }}{=}$ integer part of $x)$

$$
w(0)=1 \quad w(j)=w(j-1)+\delta([w(j-1)]) .
$$

In other words, $w \cdot T$ is the time grid to be used if we want the residence time to be constant. This new time grid is irregular in the sense that in some parts the new grid is denser than in other parts.

The reason for having $\delta$ compared with 1 and not with $T_{t}$ is because in MATLAB the index denotes the position in the vector, not the absolute time. The relation between actual sampling increment $T_{t}$ and $\delta$ is

$$
\delta \cdot T=T_{t}
$$

As mentioned previously, the time instants in the new vector are not equidistant, and since the input-output signals are not sampled at these time instants we need an estimate of the values of the signals between the sample instants. We therefore use linear interpolation between the existing values of the signals. In the sequel all parameters and variables in the resampled time will have the subscript $w$. The above described procedure is depicted in Figure 1.

Example: Assume that we have ten pairs of input-output data points with sampling time $T=1$. Let the mean value of the DIF have the value $\bar{f}=1$ and assume further that the DIF $f(t)$, the input signal $u$ (the output signal is treated analogously) and the time vector $t$ are given as follows:

$$
\begin{aligned}
& t=\left[\begin{array}{llllllllll}
1 & 2 & 3 & 4 & 5 & 6 & 7 & 8 & 9 & 10
\end{array}\right] \\
& f=\left[\begin{array}{llllllllll}
2 & 2 & 2 & 0.5 & 0.5 & 0.5 & 0.5 & 0.5 & 0.5 & 0.5
\end{array}\right] \\
& u=\left[\begin{array}{llllllllll}
2 & 4 & 6 & 8 & 10 & 8 & 6 & 4 & 2 & 0
\end{array}\right]
\end{aligned}
$$

Using resampling as described above, we will obtain the new time instant vector $w$ and the resampled input signal as

$$
\begin{aligned}
& w=\left[\begin{array}{llllllllll}
1 & 1.5 & 2 & 2.5 & 3 & 3.5 & 4 & 6 & 8 & 10
\end{array}\right] \\
& u_{w}=\left[\begin{array}{llllllllll}
2 & 3 & 4 & 5 & 6 & 7 & 8 & 8 & 4 & 0
\end{array}\right]
\end{aligned}
$$




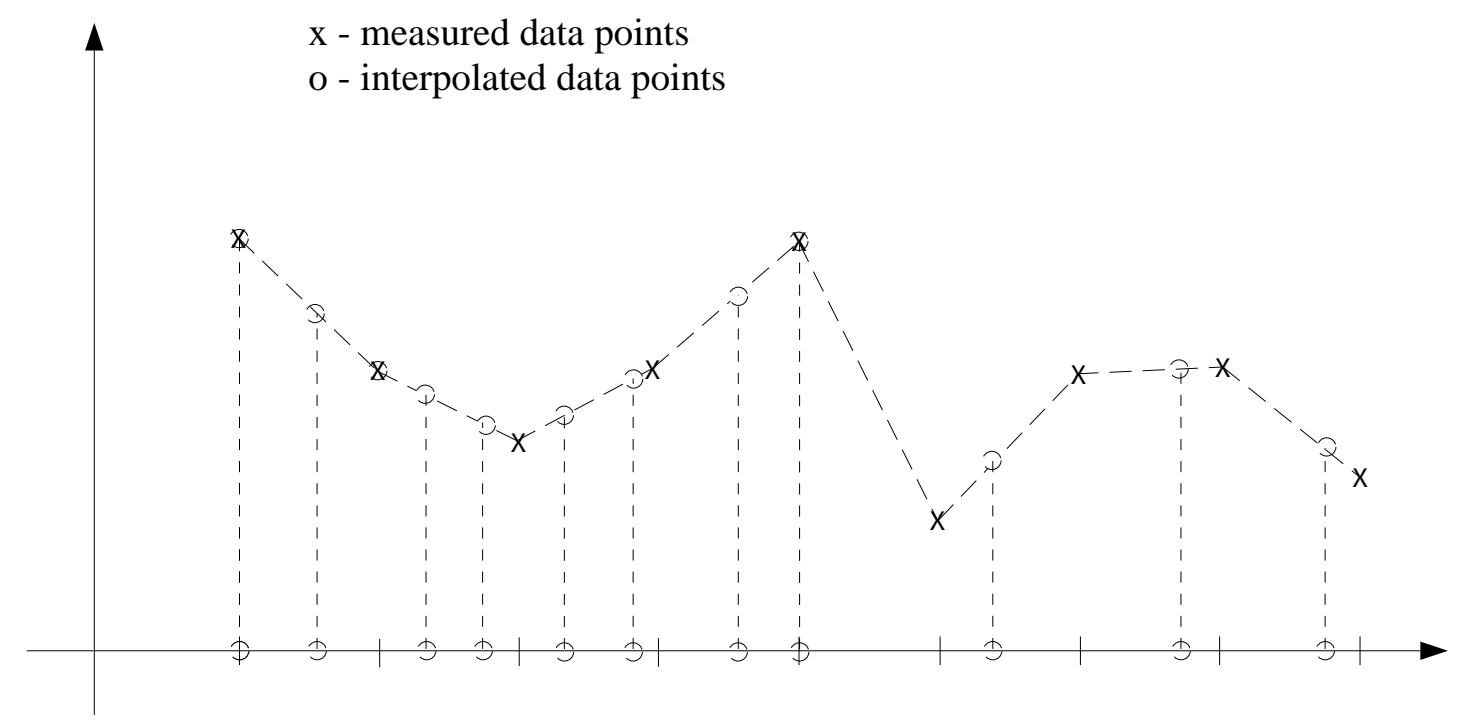

Figure 1: In the first part of the signal the dynamic influence function is high (high flow for example) so the sample increment is low, and vice versa for the second half of the signal.

The output signal is processed in the same manner as the input signal.

The computation of the vector with the new time instants $w$ can be performed in MATLAB in the following way:

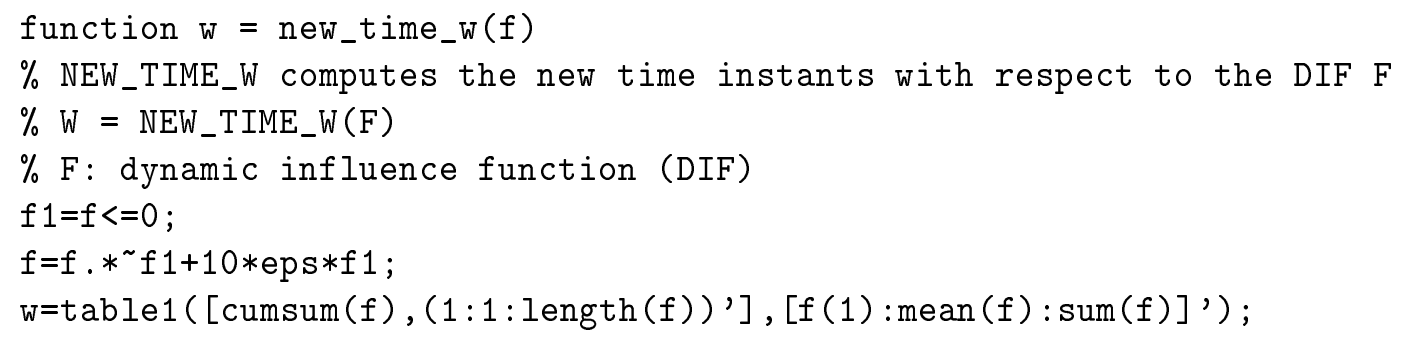

\subsection{Recursive case}

In the recursive case the problem is somewhat different. If we want the recursive algorithm to follow quick changes in the residence time (due to changes in the DIF), and at the same time be insensitive to measurement noise, we need to eliminate the influence of the DIF. The two step method, see Subsection 3.1, is used but here the first and second step are alternated during the recursive identification.

When the method is used recursively we do not know the mean DIF beforehand. The method, however, requires that it has to be known a priori. In practical cases the mean DIF is known empirically. However if that is not the case some kind of estimate of the mean value of the DIF has to be used. The better the estimate of the mean value of the DIF is, the better the obtained result will be. If a completely erroneous mean value is chosen there are two possibilities. One is that the mean value is too low, then we will sample very densely and a big part of our data will actually be interpolated data which is not preferable for use in identification. The other case is when we choose the mean value too high, then we will sample very sparsely, and hence a lot of data will be thrown away. 
Anyway, when in doubt about the mean value of the DIF underestimation of the mean is recommended. We would like to stress that the method is not sensitive to small deviations between true and estimated mean value of the DIF.

When the DIF is larger than $\bar{f}$ we also have to wait for the next value of the inputoutput signals before the interpolation can be performed. This will result in a batchlike mode of the method, since when the next measurement instant is reached, several intermediate data points will be calculated and then used in the recursive identification procedure. When the DIF is smaller that $\bar{f}$ the number of interpolated data points at every time instant is one.

\section{Reverse resampling}

\subsection{Non-recursive case}

After identification of the residence time using the resampled signals, the resampling has to be reversed to obtain the actual value of the residence time, i.e., in hours instead of samples. To reverse the resampling we have to use the DIF again and the residence time (in hours) is calculated for every sampling instant as

$$
\tau(i)=\frac{\bar{f}}{f(i)} \cdot \hat{\tau}_{w} \cdot T
$$

where $T$ is the sampling time and $\hat{\tau}_{w}$ is the residence time identified with the resampled input-output signals. This can also be extended to take care of the recursive identification case. It will, however, be more complicated. This routine can be written in MATLAB as

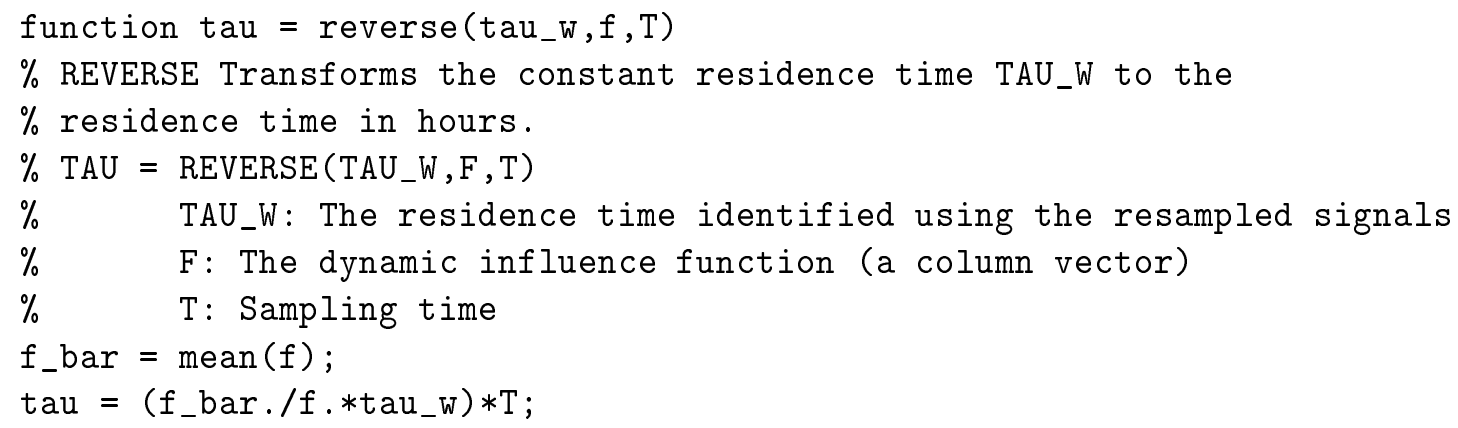

\subsection{Recursive case}

When recursive identification of the residence time has been performed, we will have the residence time $\hat{\tau}_{w}(i)$ at every sampling instant corresponding to the $w$-vector. We, however, want to have the residence time $\hat{\tau}(i)$ given in the original time instants. This can be performed by interpolation in the $\hat{\tau}_{w}$-vector. When the interpolation has been performed, the mean value of the dynamical influence function $\bar{f}$ and the DIF $f(i)$ is used to compute the residence time in hours as

$$
\tau(i)=\frac{\bar{f}}{f(i)} \cdot \hat{\tau}(i) \cdot T
$$

The calculation in (8) can be implemented in MATLAB in the following way 


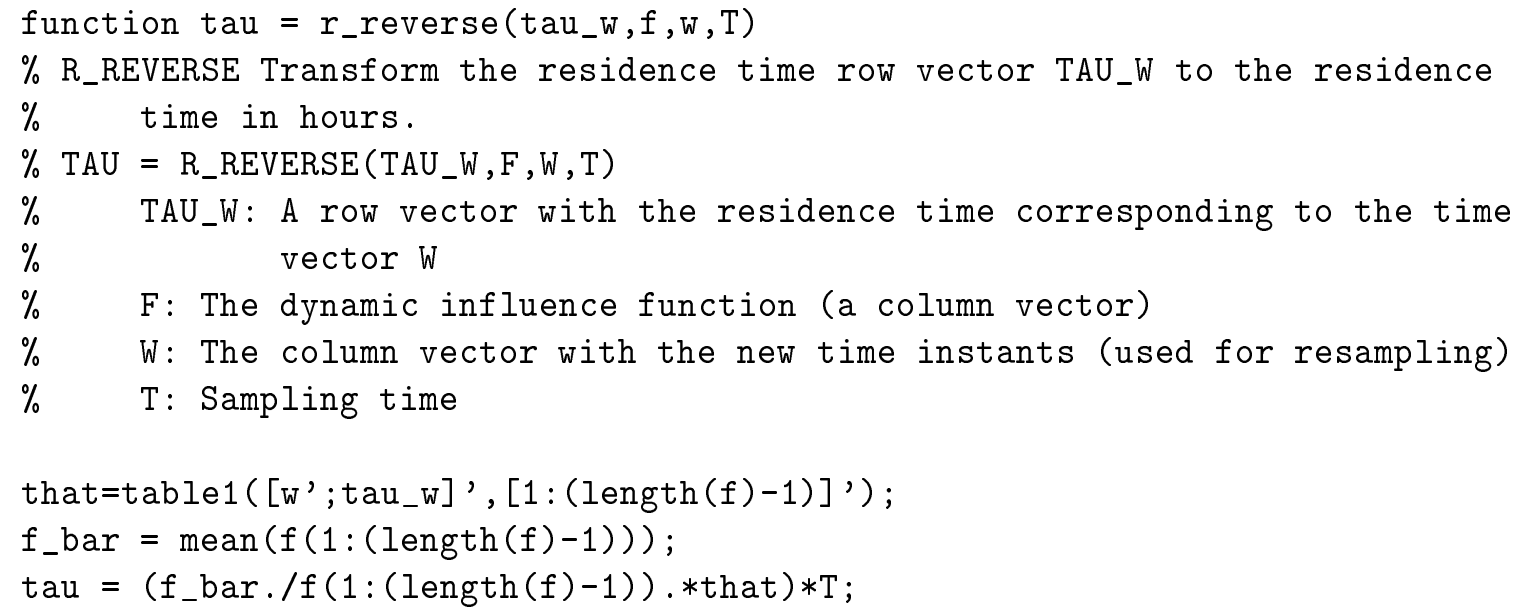

\section{Example}

In pulp processes there are often quick changes in residence time due to changes in pulp flow and we will therefore use a sub-plant of a pulp plant to exemplify the previously described method. The sub-system contains a washing plant with two blending chests and a buffer tank, see Figure 2. Here the largest part of the residence time originates from the buffer tank.

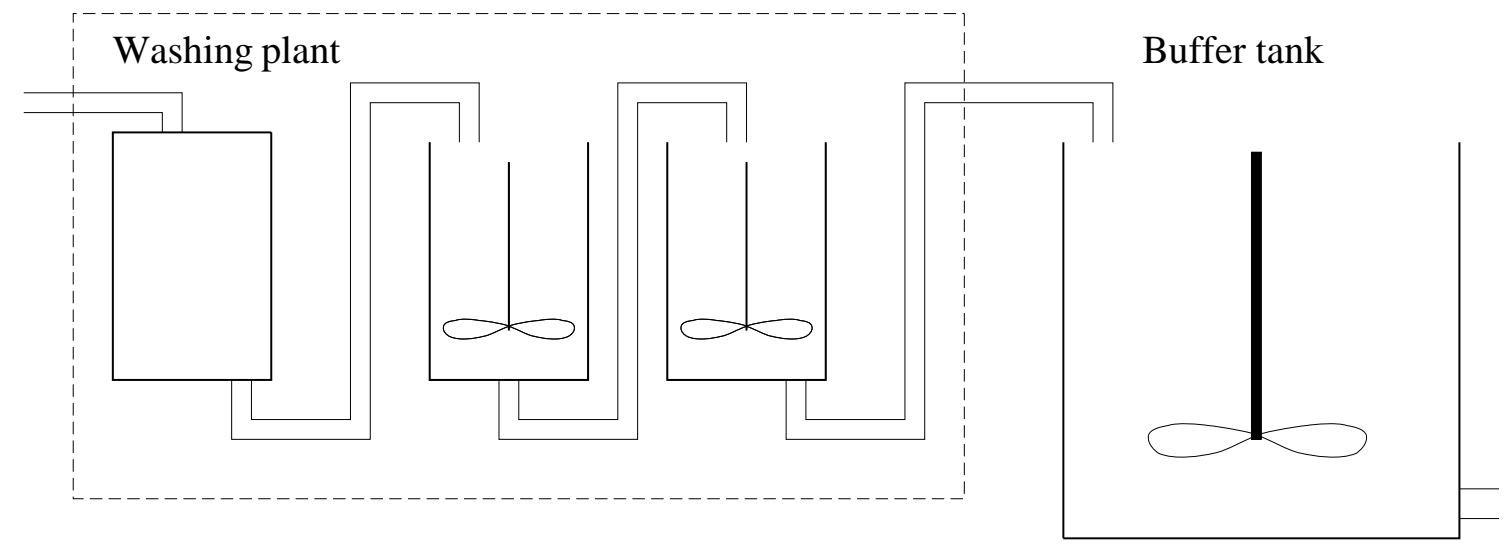

Figure 2: The sub-system of the pulp plant.

Since we are interested in the residence time the input-output signals have to be highly dependent of the pulp flowing through the system. In this special case a quality variable, the kappa number, a measure of the amount of lignin in the fibers, is suitable. The kappa number will be sampled before the washing plant and after the buffer tank with a sampling rate of $1 / 12$ minutes. For this system the residence time is obviously at least influenced by two variables, the pulp flow and the level of the buffer tank. Here we use the measured level instead of the volume of the tank since they differ only by a constant factor due to the cylindrical shape of the tank. In this case the DIF will therefore be chosen as flow/level, which is inversely proportional to the residence time, that is

$$
f(t)=\frac{\text { flow }}{\text { level }}(t) .
$$

Inital measurements (tracer-tests) showed that the sub-plant in question could be modeled by two types of time delays and a dynamic system without time delay, coupled 


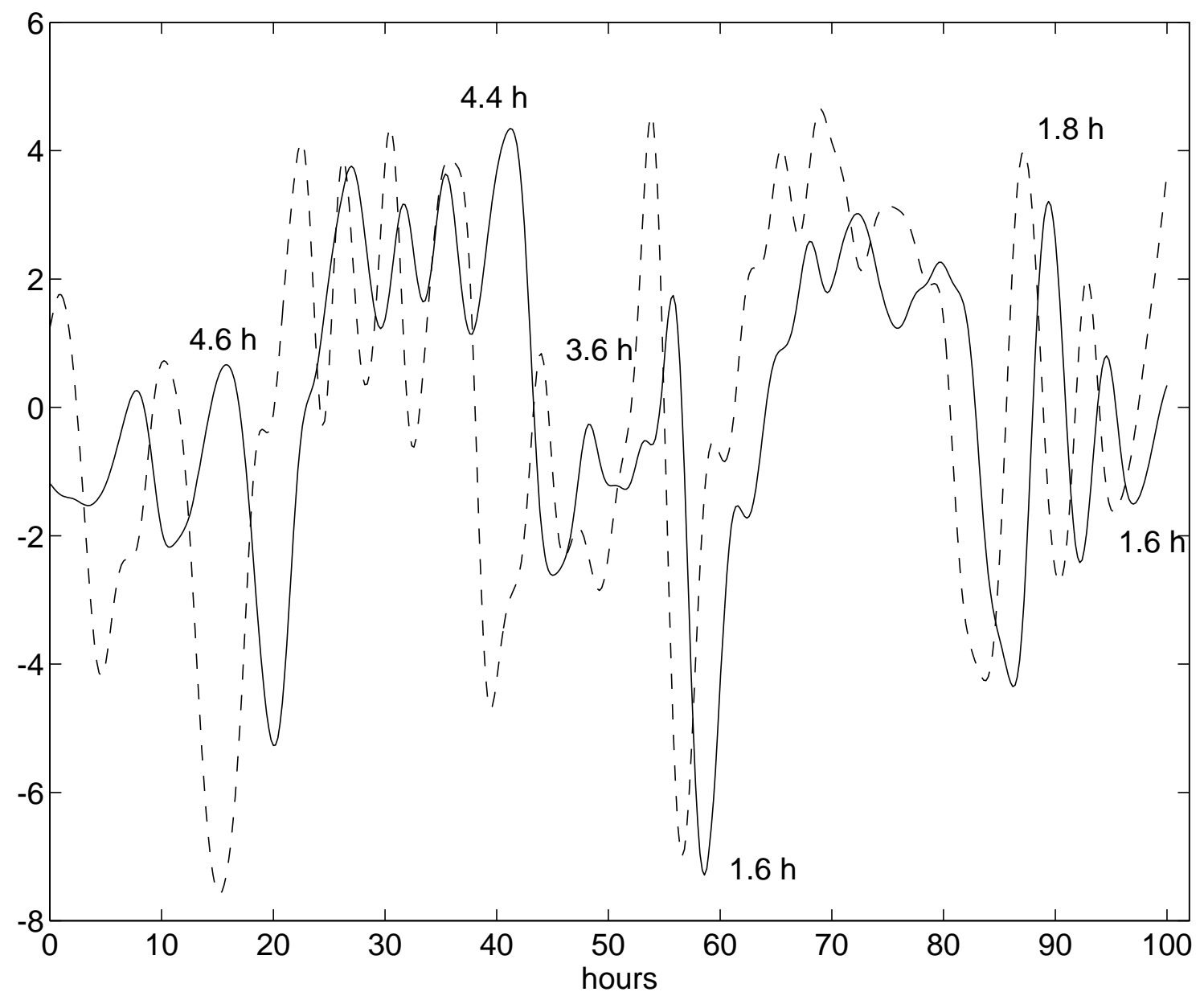

Figure 3: Input- (dashed) and output (solid) kappa numbers, where the by inspection estimated residence times also are depicted in the figure.

in series. The first time delay is due to the inital washing part (function of the flow), see Figure 2, and the second time delay is due to the fact that the large tank behaves like a pure time delay and a dynamic system in series. The time delay in the large tank, however, depends on both the flow and the level in the tank. Note that the algorithm as presented so far is applicable to systems with none or constant time delay. We will, however, in the example below which has time varying time delay, apply the algorithm with no modifications, and comment the result at the end of this section.

\subsection{Non-recursive case}

If we now study the measured input-output signals, e.g. the kappa numbers, we can by inspection in Figure 3 see that the residence time is varying between approximately 1.8 hours to 4.6 hours. It should be noted that the signals in Figure 3 also are lowpass filtered.

In Figure 4 the DIF $f=$ flow/level is depicted. The pulp flow and the level of pulp in the buffer tank can be seen in Figure 5 and Figure 6 respectively. The DIF has been processed so that no numerical problems will occur. The production stops (flow $\leq 0$ ) have been replaced with a very low production to prevent overflow in time increment $T_{t}$.

The input-output signals are then resampled using the DIF $f$, and the resulting inputoutput signals can be seen in Figure 7 where we by inspection see that the variation has 
Flow/Level

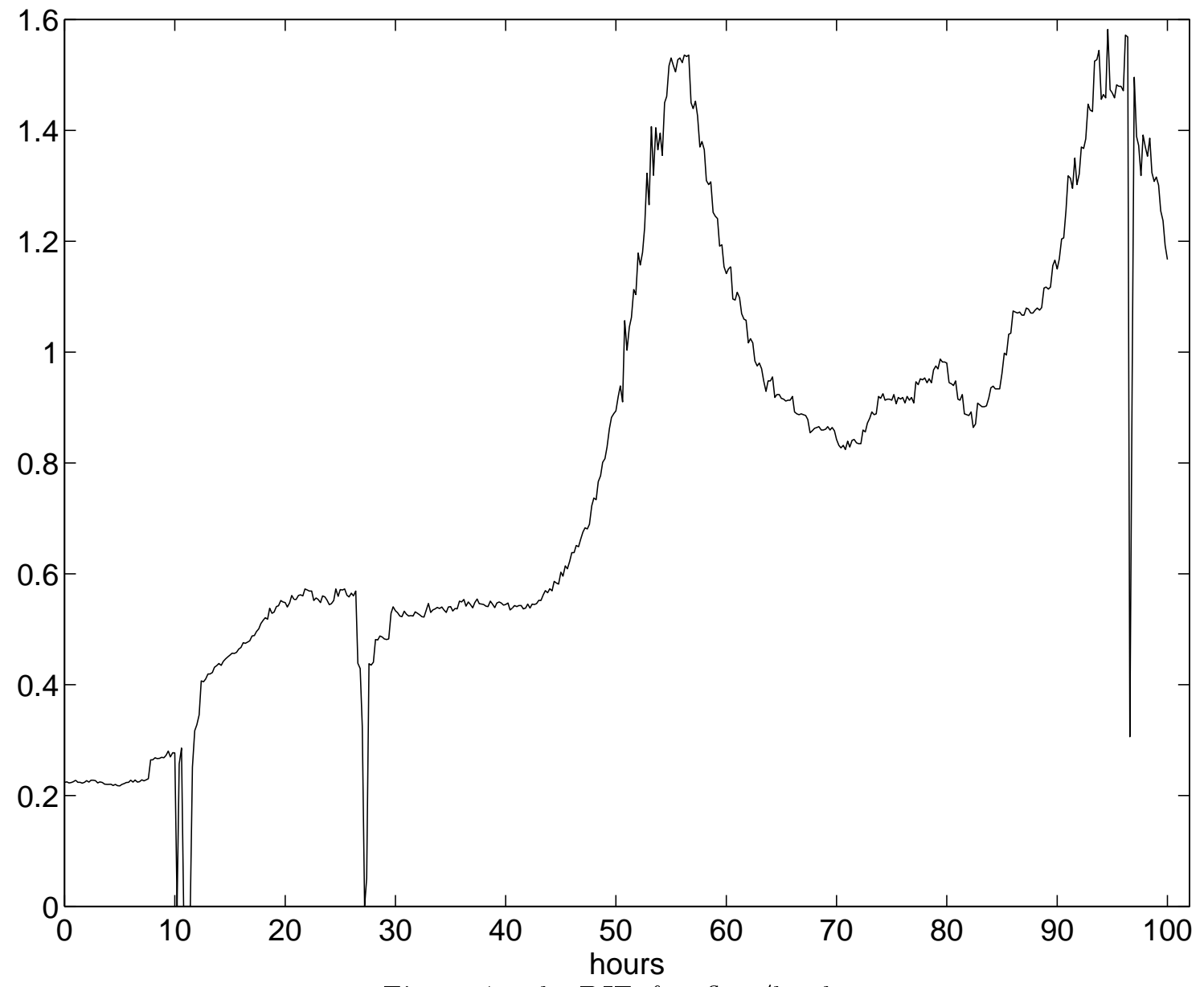

Figure 4: The DIF $f=$ flow/level 
Flow

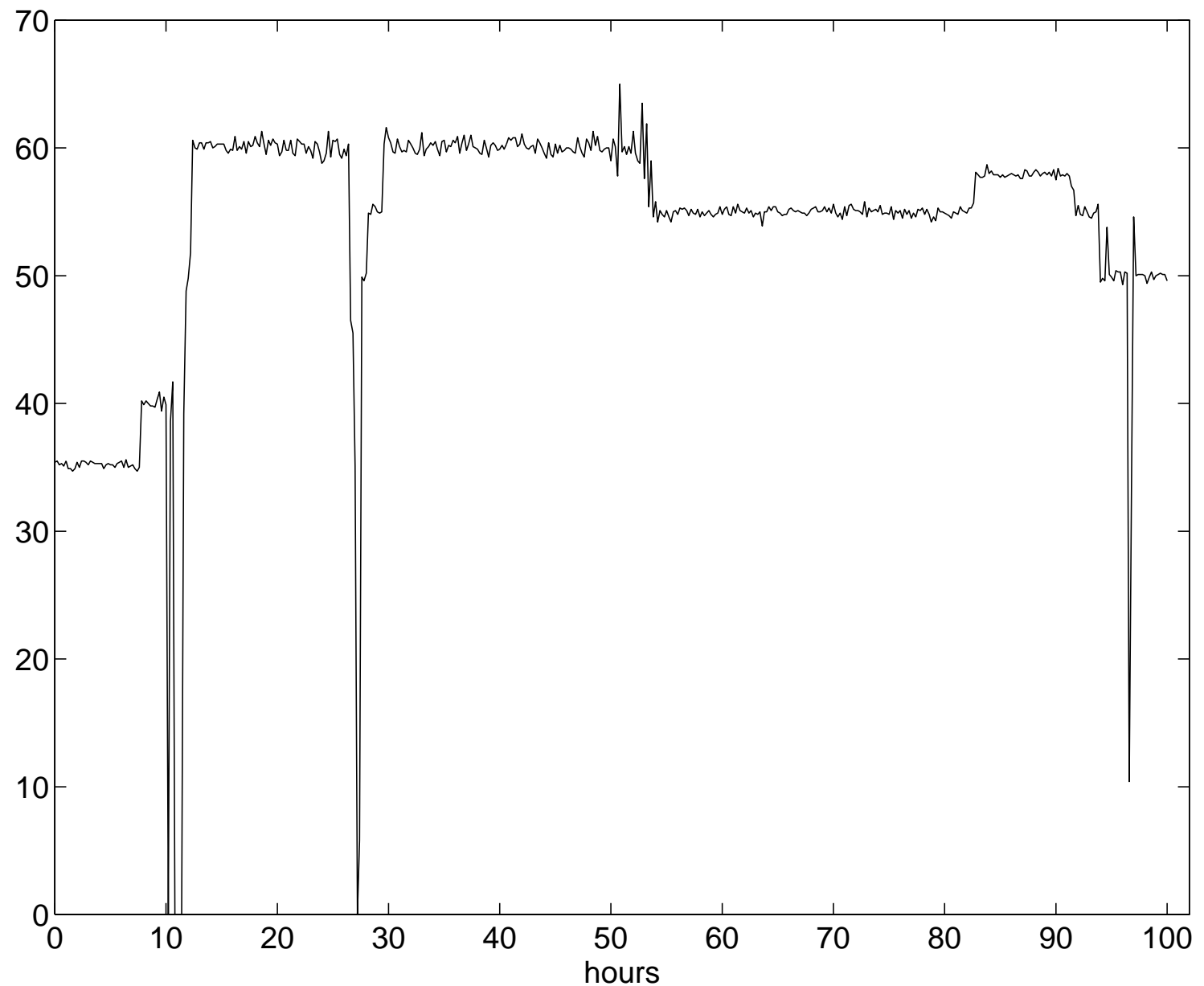

Figure 5: Flow through the system.

been decreased with a factor 4 .

We are now able to use ordinary identification methods to obtain a suitable model. In this special example the used models are restricted to be of ARX type, see [3].

Example An ARX-model of second order can be written as

$$
y(t)=-a_{1} y(t-T)-a_{2} y(t-2 T)+b_{1} u\left(t-n_{k} T\right)+b_{2} u\left(t-\left(n_{k}+1\right) T\right)+e(t)
$$

This expression can also be written on the regression form (3).

In the identification process, using the resampled signals, it turned out that the best model was an ARX-model of second order and eleven samples pure time delay. To validate an ARX-model we compare a simulated output signal with a measured one, using a new independent data set. Such a simulation, which is performed with resampled data, is shown in Figure 8. In the simulation we see the measured output signal (dashed) and the simulated one (solid). We see that the simulated signal follows the measured signal in a satisfactory way. What would the result have been if we had not resampled the signals? If we estimate an ARX-model with the original input-output signals (without resampling the signals) the simulation will be as in Figure 9. We stress that this ARX-model is not the same as the previous one estimated on resampled signals. It is the best one we could find in the sense that the simulation results using it are the best. We see that the simulated 


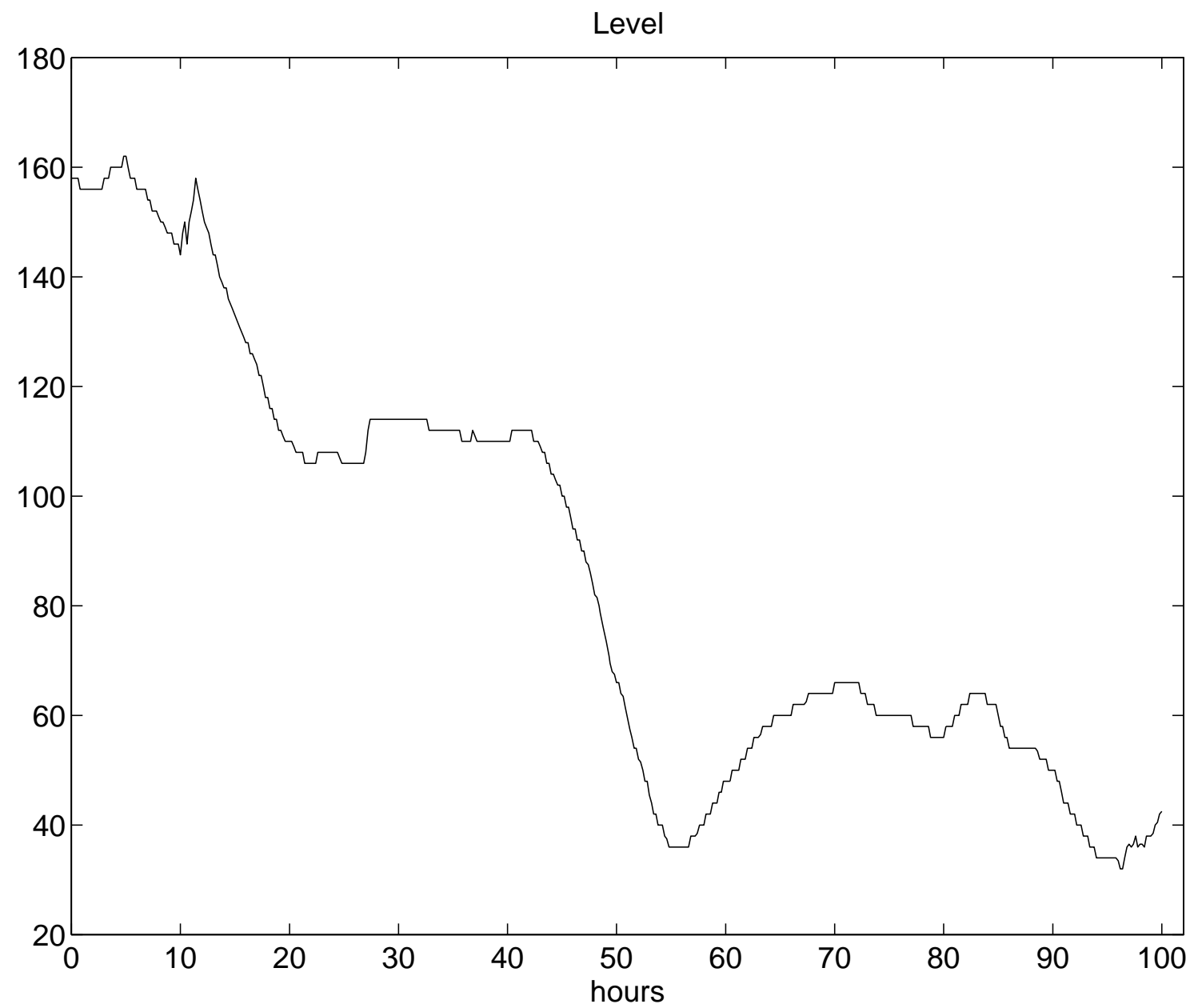

Figure 6: Level in the tank. 


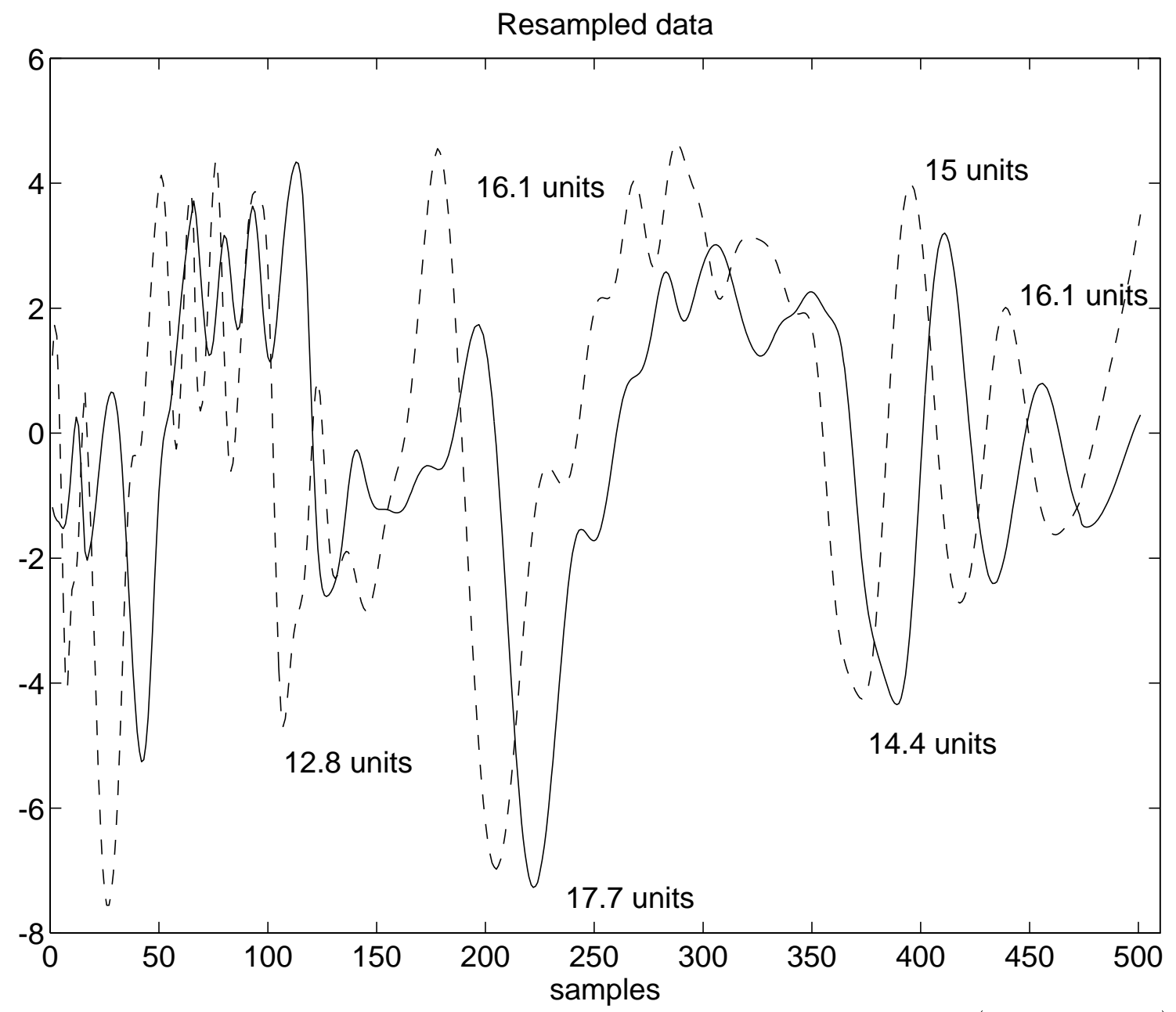

Figure 7: Resampled input-output kappa numbers where the estimated (by inspection) residence times are marked out. 


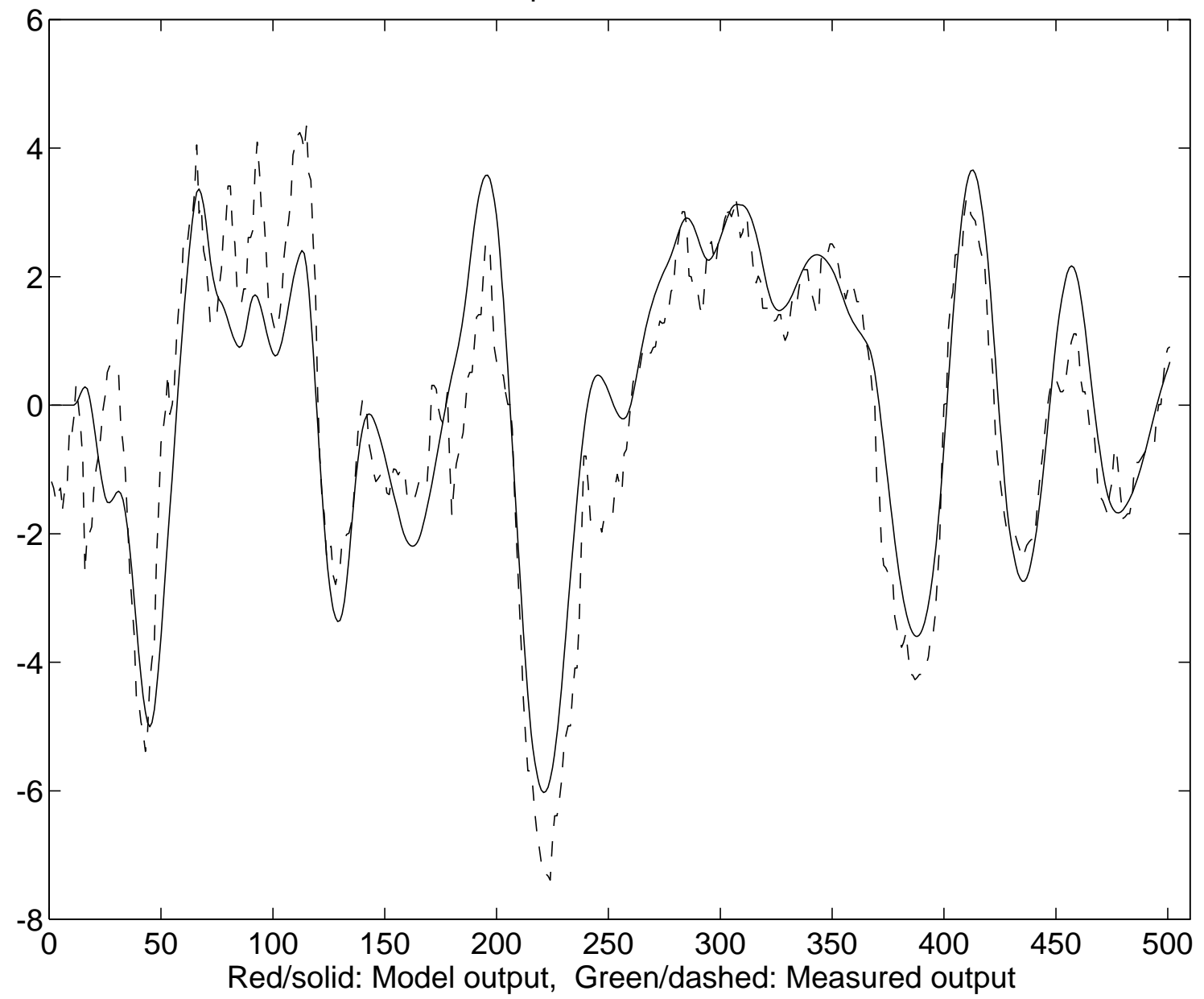

Figure 8: Simulated output kappa number(solid) compared with the real output kappa number (dashed). The model is obtained with resampled signals

signal does not follow the measured signal nearby as good as in the previous case. The resampling thus considerably improves the identification of the model.

The simulations thus show that the resampling followed by identification considerably improves the "simulation ability" of the obtained model, if compared to identification without resampling. In fact, the simulation ability of the model is the most revealing characterstic concerning the model quality. This suggests that in this example the time delay varies in such a way that the algorithm works well. The investigation of different kinds of models of time varying delays is in progress. This example suggests that the presented algorithm may be useful also in cases where all the preconditions are not fully met.

\subsection{Recursive case}

Despite the resampling, there are fluctuations in the residence time and this can be taken care of using recursive identification, see [5]. In Figure 10 the recursive identification of residence time, on data in Figure 7, is shown (the value of the forgetting factor is 0.97 ). In Figure 10 the unit for residence time is sample, so what we see is the fluctuation of residence time in the resampled signals. The fact that residence time fluctuates even though the signals are resampled, is caused by that the DIF used does not contain the 
Output \# 1 Fit: 1.677

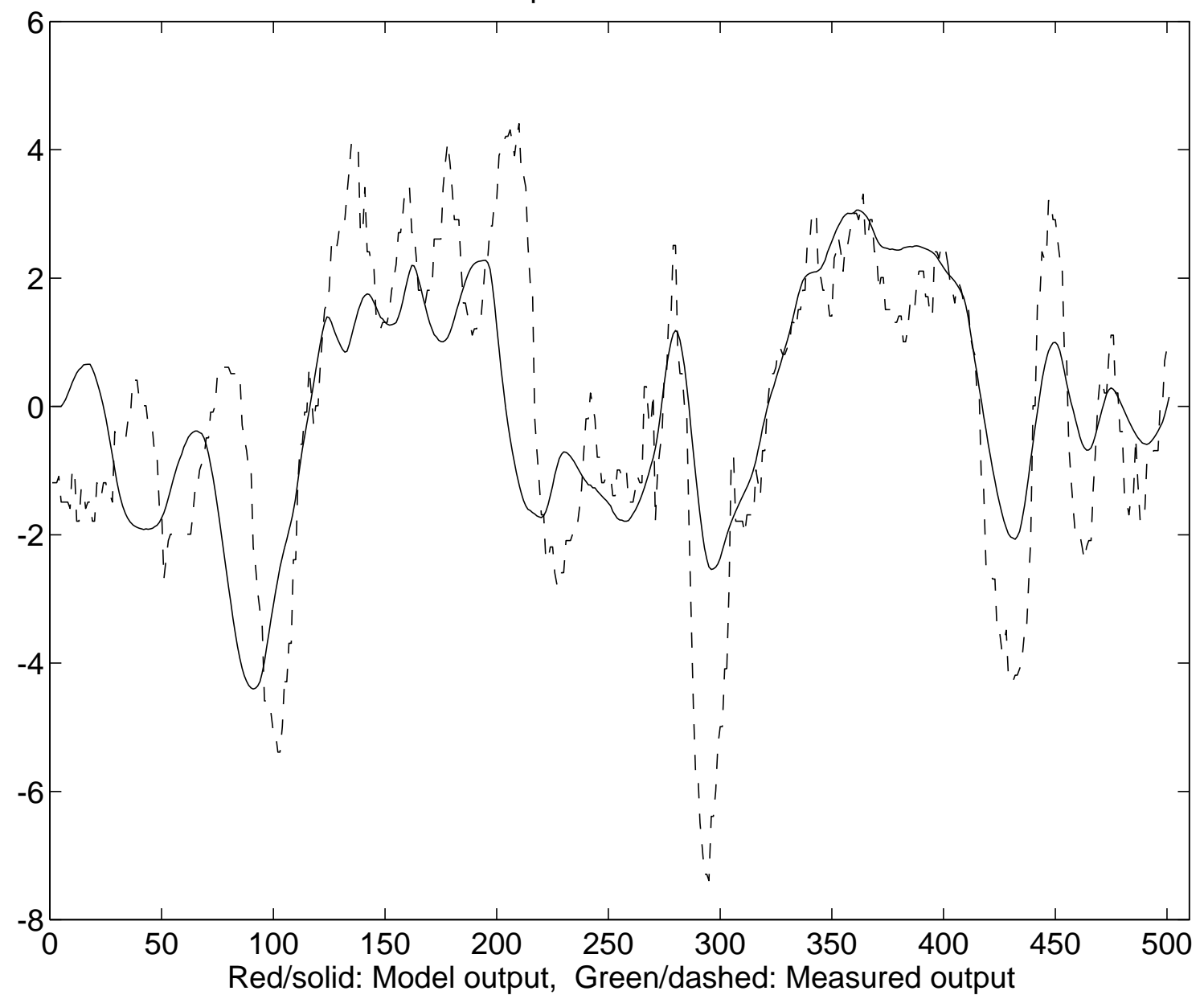

Figure 9: Simulated output (solid) compared with the real output (dashed). The model is obtained without resampling the signals 


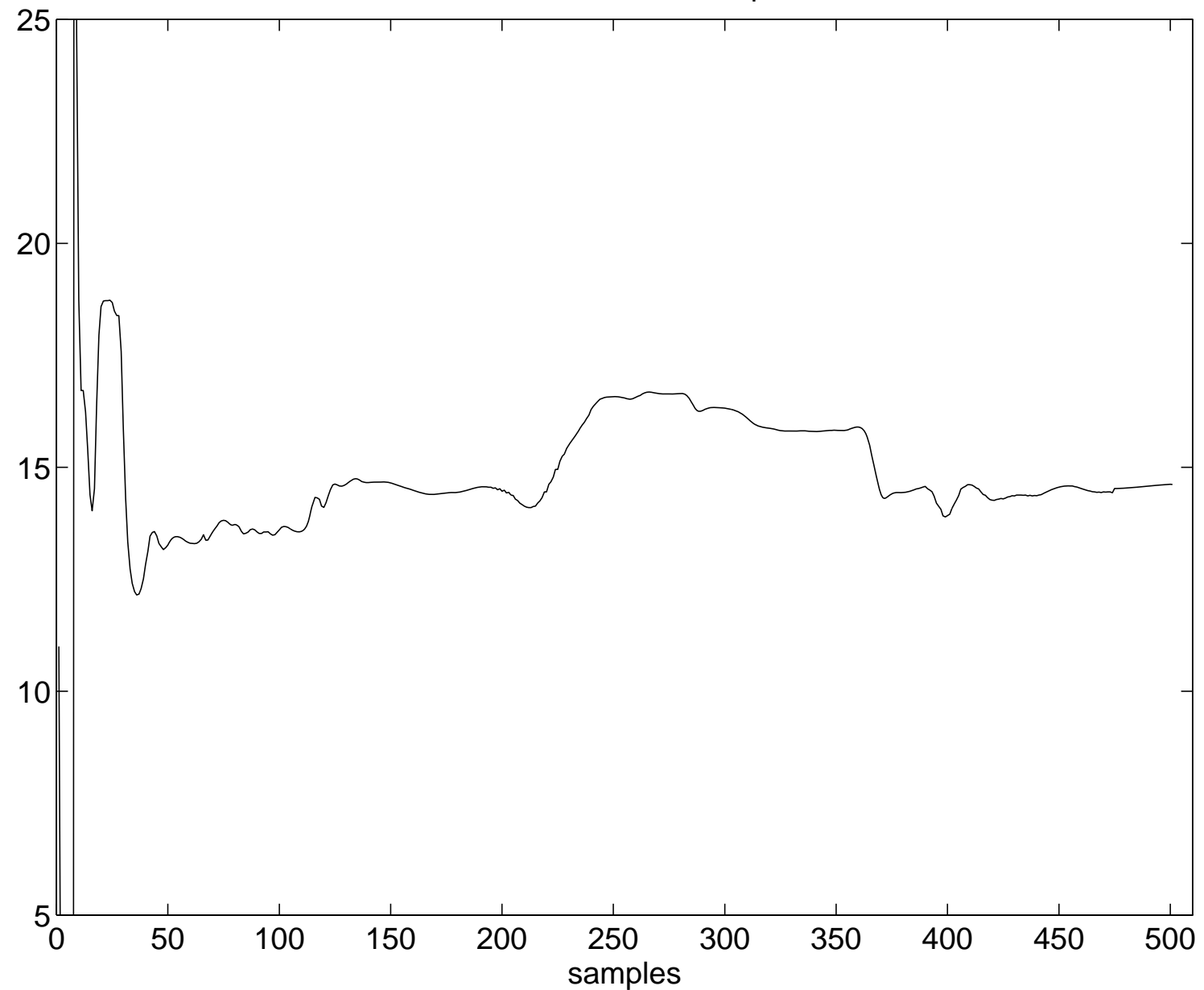

Figure 10: Recursive identification of residence time

complete information about the time variability. To get the residence time (in hours) we have to reverse the resampling, the procedure is described in section 4.2.

In Figure 11, the residence time (in hours) for the process estimated by recursive identification (dashed) and the off-line estimate (solid) are shown. Here we have reversed the resampling using the method in Section 4.2. We conclude this example by showing, in Figure 12, estimates by recursive identification without applying resampling on data in Figure 3 with forgetting factor 0.995 (dashed), forgetting factor 0.97 (dotted) and the off-line estimate with resampling (solid). We see that by decreasing the forgetting factor the estimate gets closer to the off-line estimate. The estimate, however, becomes more "noisy".

All numerical computations in this example were performed in MATLAB using the System Identification Toolbox, see [4].

\section{Summary}

A method for estimation of residence time in continuous flow systems with varying dynamics has been presented. The proposed method consists of two steps; in the first step the time variability is reduced by resampling the signals and in the second step an ordinary identification method is applied. The residence time in hours is then obtained by reversing 
Residence time (in hours)

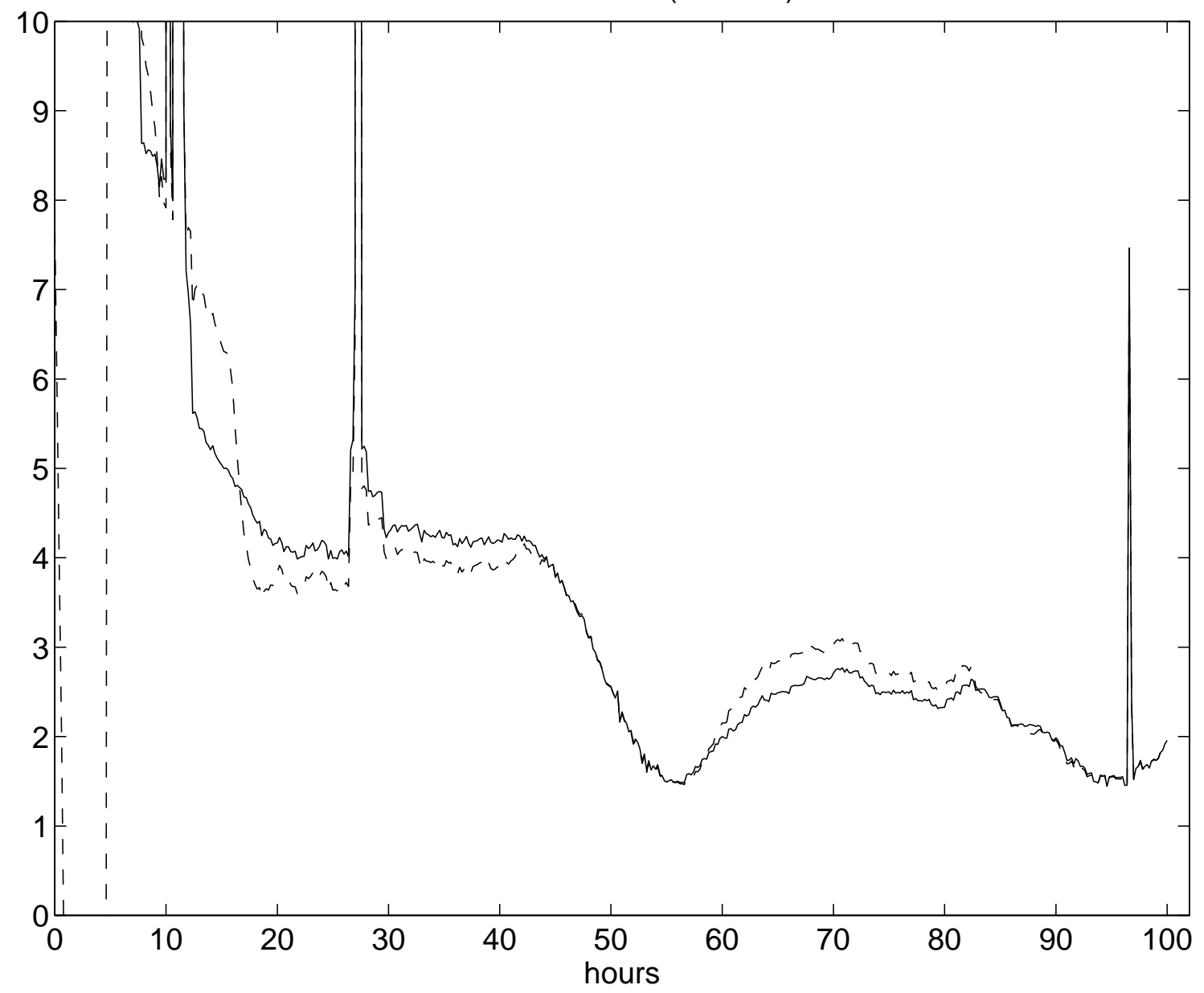

Figure 11: The residence time in hours estimated by recursive identification (dashed) and off-line identification (solid). 
Residence time (in hours)

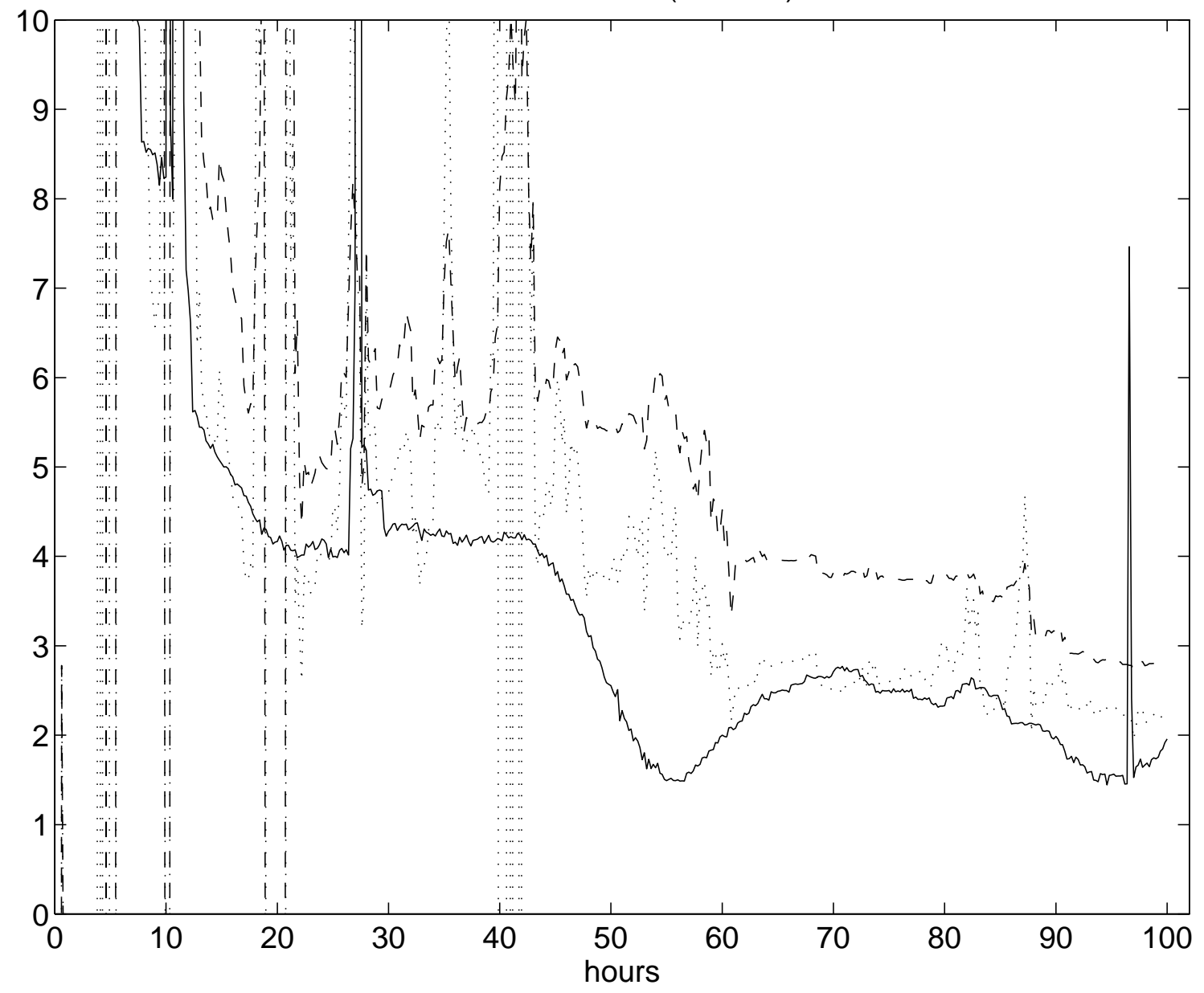

Figure 12: The residence time in hours estimated by recursive identification without applying resampling with forgetting factor 0.995 (dashed), with forgetting factor 0.97 (dotted) and the off-line estimite with resampling (solid) 
the resampling.

The method has also been used together with recursive identification as an improvement of tracking ability of an ordinary recursive routine. Assume a recursive least squares algorithm with forgetting factor is chosen for estimation of the model. If the goal is to track abrupt changes in the residence time, due to e.g. flow changes, a low forgetting factor has to be chosen. This means that the estimate will be very "noisy" since the algorithm is sensitive to measurement noise. If the signals are resampled according to the presented method, a high forgetting factor can be chosen and the recursive algorithm can concentrate to track the slow changes of residence time, due to e.g. flow pattern changes etc.

\section{References}

[1] J. Harmon, P. Pullammanappallil, S.A. Svoronos, G. Lyberators, and D.P. Chynoweth. On-line identification of a variable sampling period discrete model and its use for the adaptive control anaerobic digestion. In Proc. $A C C$, volume 2, pages 1540-1545, 1990.

[2] A. Isaksson. Estimation of average residence time given an identified arx-model. Technical report lith-isy-i-1422, Department of Electrical Engineering, Linköping University, Sweden, 1993.

[3] L. Ljung. System Identification: Theory for the User. Prentice-Hall, Englewood Cliffs, NJ, 1987.

[4] L. Ljung. System Identification Toolbox-User's Guide. The Math Works Inc., 1991.

[5] L. Ljung and T. Söderström. Theory and Practice of Recursive Identification. MIT Press, Cambridge, Massachusetts, 1983.

[6] L.Tian, A.J.Niemi, and R.Ylinen. Recursive process identification under variable flow and volume. In Preprints of the 3rd IFAC Symposium on Dynamic and Control of Chemical Reactors, Distillation Columns and Batch Processes, 1992.

[7] A. J. Niemi. "Residence Time Distributions of Variable Flow Processes". International Journal of Applied Radiation and Isotopes, 28:855-860, 1977.

[8] J.S. Shamma and M. Athans. "Guaranteed Properties of Gain Scheduled Control for Linear Parameter-varying Plants". Automatica, 27(3):559-564, 1991.

[9] T. Wigren. Recursive Identification Based on the Nonlinear Wiener Model. PhD thesis, Uppsala University, Sweden, 1990.

[10] K. Zenger. Analysis and control design of a class of time-varying systems. Report: 88, Control Engineering Laboratory, Helsinki University of Technology, 1992. 